\title{
The Role of Zinc in Depressed Pregnant and Non-Pregnant Women: A Systematic Review and Meta-Analysis
}

\author{
Nadine B. Page ${ }^{1}$, Anna M. Brown ${ }^{1}$, Erin Pascoe ${ }^{2}$, Sally Braithwaite ${ }^{3}$, Michelle L. Townsend ${ }^{4}$, \\ Jane S. Herbert ${ }^{4}$, Hilary Davies ${ }^{1}$, Kathryn H. Hart ${ }^{1}$ and J. Bernadette Moore \\ ${ }^{1}$ University of Surrey, Guildford, United Kingdom, \\ ${ }^{2}$ St. Peter's Hospital NHS Foundation Trust, Chertsey, United Kingdom, \\ ${ }^{3}$ Surrey and Borders Partnership NHS Foundation Trust, Guildford, United Kingdom, \\ ${ }^{4}$ University of Wollongong, Wollongong, Australia and \\ ${ }^{5}$ University of Leeds, Leeds, United Kingdom
}

\begin{abstract}
Perinatal depression is a depressive illness that affects $10-15 \%$ of women in the UK with an estimated cost of $£ 1.8$ billion/year. Zinc deficiency is associated with the development of mood disorders and zinc supplementation has been shown to help reduce the symptoms of depression. Women who are pregnant and breastfeeding are at risk of lower levels of zinc because of the high demand from the developing and feeding baby. However, studies in the perinatal period are limited. With a long-term aim of designing a randomised controlled trial (RCT) to examine if zinc supplementation reduces depressive symptoms in pregnant and lactating women; the objective of this review was to systematically evaluate previous RCTs assessing zinc supplementation and depressive symptoms, in order to establish a zinc dosing regimen with regards to Galenic formulation, unit dose and frequency. The review was conducted by independent reviewers in accordance with PRISMA guidelines and is registered at Prospero (CRD42017059205). The Allied and Complimentary Medicine, CINAHL, Embase, MEDLINE, PsycINFO, PubMed, and Cochrane databases were searched since records began, with no restrictions, for intervention trials assessing Galenic formulation, unit dose and frequency of zinc supplementation to reduce the symptoms of depression. From a total of 66 identified records, 7 articles met the inclusion and exclusion criteria; all assessed the effect of zinc supplementation on mood. Risk of bias was independently assessed using the standard 'Cochrane risk of bias tool'. Overall, 5 of the 7 papers were rated as high-quality trials; of the other two, one was rated poor and the other fair but both had a number of learning points. Preliminary findings indicate at the end of supplementing zinc, depression scores were reduced significantly. In one study, the Beck score decreased in the placebo group, but this reduction was not significant compared to the baseline. In two of the studies there was a significant correlation between serum zinc and self-reported mood questionnaires. Results also suggest that $25 \mathrm{mg}$ zinc supplementation combined with antidepressant drugs can be effective in the treatment of major depression in women. This supports other work where researchers supplemented $25 \mathrm{mg}$ of elemental zinc for 12 weeks or longer and found a reduction of symptoms in both pregnant and non-pregnant women. Thus, an early conclusion is that $25 \mathrm{mg}$ of elemental zinc is an effective dose for improving low mood and is achievable in a trial setting.
\end{abstract}

\section{Conflict of Interest}

There is no conflict of interest 